\title{
A novel device for chronic intracranial drug delivery via microdialysis
}

\author{
T.J. Bazzett ${ }^{1,2}$, J.B. Becker ${ }^{1}$ and R.L. Albin ${ }^{2}$ \\ ${ }^{\prime}$ Department of Psychology, Neuroscience and Reproductive Sciences Programs and ${ }^{2}$ Department of Neurology, Neuroscience \\ Program, The University of Michigan, Ann Arbor, MI 48104-1687 (U.S.A.)
}

(Received 7 March 1991)

(Accepted 28 June 1991)

Key words: Microdialysis; Chronic intracranial infusion; Neural degeneration; Striatum; Osmotic minipump; Quinolinic acid

\begin{abstract}
A system is described for chronic intracranial drug administration in the rat using a modified in vivo microdialysis probe coupled to an Alzet model 2002 osmotic minipump. The results presented demonstrate that this system can be used for the chronic administration of quinolinic acid with minimal non-specific damage. Each pump delivered approximately $225 \mu \mathrm{l}$ of solution over a period of 19-20 days when tested in vitro. The dialysis units were uniform in function, delivering $>93 \%$ of the [ $\left.{ }^{3} \mathrm{H}\right] \mathrm{quinolinic}$ acid initially loaded into the minipump. For in vivo analysis of this apparatus the dose of quinolinic acid tested produced extensive destruction of the striatum. The present system allows reliable drug diffusion over a relatively large area without pressure injection variability. In conclusion, we have developed a simple and inexpensive technique for administration of drugs into brain parenchyma with substantial advantages over previously used techniques.
\end{abstract}

\section{Introduction}

Chronic drug administration in vivo has been demonstrated repeatedly using Alzet osmotic minipumps implanted either subcutaneously or intraperitoneally (Nielsen, 1981; Mendel et al., 1988; Rivier and Vale, 1989; Sasamura et al., 1990). Osmosis of extracellular fluid into the pump displaces the fluid within an internal reservoir and causes slow release of the solution contained within the pump. Drug administration by this method can be local, at the site of the pump,

Correspondence: Dr. T.J. Bazzett, Neuroscience Laboratory Building, 1103 E. Huron St., Ann Arbor, MI 48104-1687, U.S.A. or distal if a section of flexible tubing is attached. For example, several researchers have reported successful intracranial infusion of drugs by attachment of a subcutaneous pump to a chronically implanted intracranial cannula (Mangano and Schwarcz, 1983; McBean and Roberts, 1984; Hargraves and Freed, 1987; Rieke et al., 1989).

This method of administration is generally uncomplicated for intraventricular infusion. There are problems, however, with using this technique for chronic infusion into brain parenchyma. Cannulae provide only a point source of drug, and this delivery method may produce non-specific damage due to the volume of fluid introduced. Furthermore, while some drugs may diffuse freely through tissue, unique properties of other substances limit diffusion to a small region surround- 
ing the cannula tip, thus limiting the area affected by drug infusion (Evans et al., 1975). Finally, incomplete expulsion of fluid by the pump, possibly caused by cannula obstruction, has also been reported (Hargraves and Freed, 1987).

To reduce problems associated with chronic infusion using intracranial cannulae, a novel apparatus was developed that uses a modified in vivo microdialysis probe coupled with an Alzet minipump. The present report describes the successful in vitro and in vivo use of this apparatus to deliver the excitotoxin, quinolinic acid (QUIN), to the striatum.

\section{Methods}

\section{Probe construction}

The design of the chronic microdialysis apparatus is shown schematically in Figs. 1 and 2 with dimensions for intrastriatal administration. The microdialysis section of the apparatus is adapted from an in vivo microdialysis probe (Robinson and Camp, 1991). The cost of materials is less than $\$ 7.50$ plus the cost of the osmotic minipump. The assembly is constructed over 3 days, allowing epoxy compounds to dry overnight.

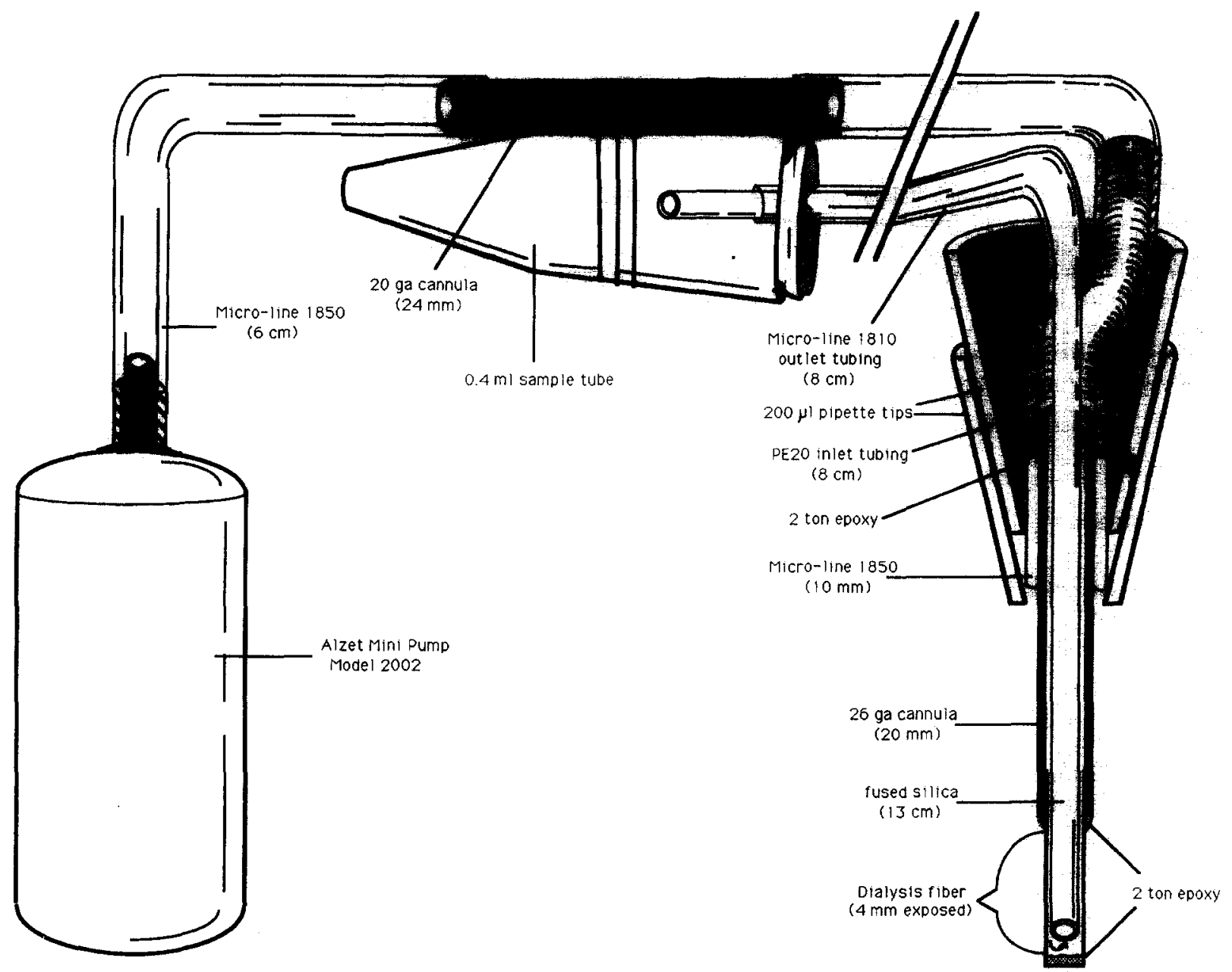

Fig. 1. A schematic illustration of the components used to construct a chronic microdialysis apparatus designed for intrastriatal delivery in the adult rat. 
Step 1. A $20-\mathrm{mm}$ length of 26-ga thin wall stainless steel tubing is cut; the ends and lumen are cleaned. The cannula is then acid etched to facilitate epoxy adhesion using the following procedure: (1) degrease in xylene for $5 \mathrm{~min},(2)$ acid pickle in $4 \% \mathrm{H}_{2} \mathrm{SO}_{4}, 4 \% \mathrm{HCl}$ in water for 20 min, (3) acid etch in $12 \% \mathrm{HNO}_{3}, 2 \%$ hydrofluoric acid in water for $15 \mathrm{~min}$, and (4) rinse in water then in methanol, and dry.

A 10-12-mm length of dialysis fiber is cut with iris scissors, taking care that the end has not been crimped. Using a fine pair of watchmaker forceps to grasp one end, the dialysis fiber is inserted into one end of the stainless steel cannula with 7-8$\mathrm{mm}$ protruding. Using a no. 00 insect pin, a small amount of 2-ton epoxy is applied around the circumference of the dialysis fiber adjacent to the cannula. The dialysis fiber is tapped further into the cannula so that the epoxy adheres to the inside of the cannula. The protruding dialysis fiber is cut to $4.25 \mathrm{~mm}$ from the epoxy 'collar.' A small drop of epoxy is applied to the end of the dialysis fiber to close the end. Capillary action should draw the epoxy approximately $0.25 \mathrm{~mm}$ into the fiber. Allow to dry overnight.

Step 2. A 13-cm length of fused silica capillary tubing is cut using a diamond edge cutter. Using a 30-ga needle, the fused silica tubing is drawn through the side wall and out one end of an 8-cm length of PE20 tubing (Fig. 1.) The fused silica is inserted into the open end of the stainless steel/dialysis fiber piece made in step 1 and pushed through the stainless steel cannula into the dialysis fiber $(0.25 \mathrm{~mm}$ from the end $)$. The PE20 tubing is pressure fit over the end of the cannula and the exposed end of the fused silica is

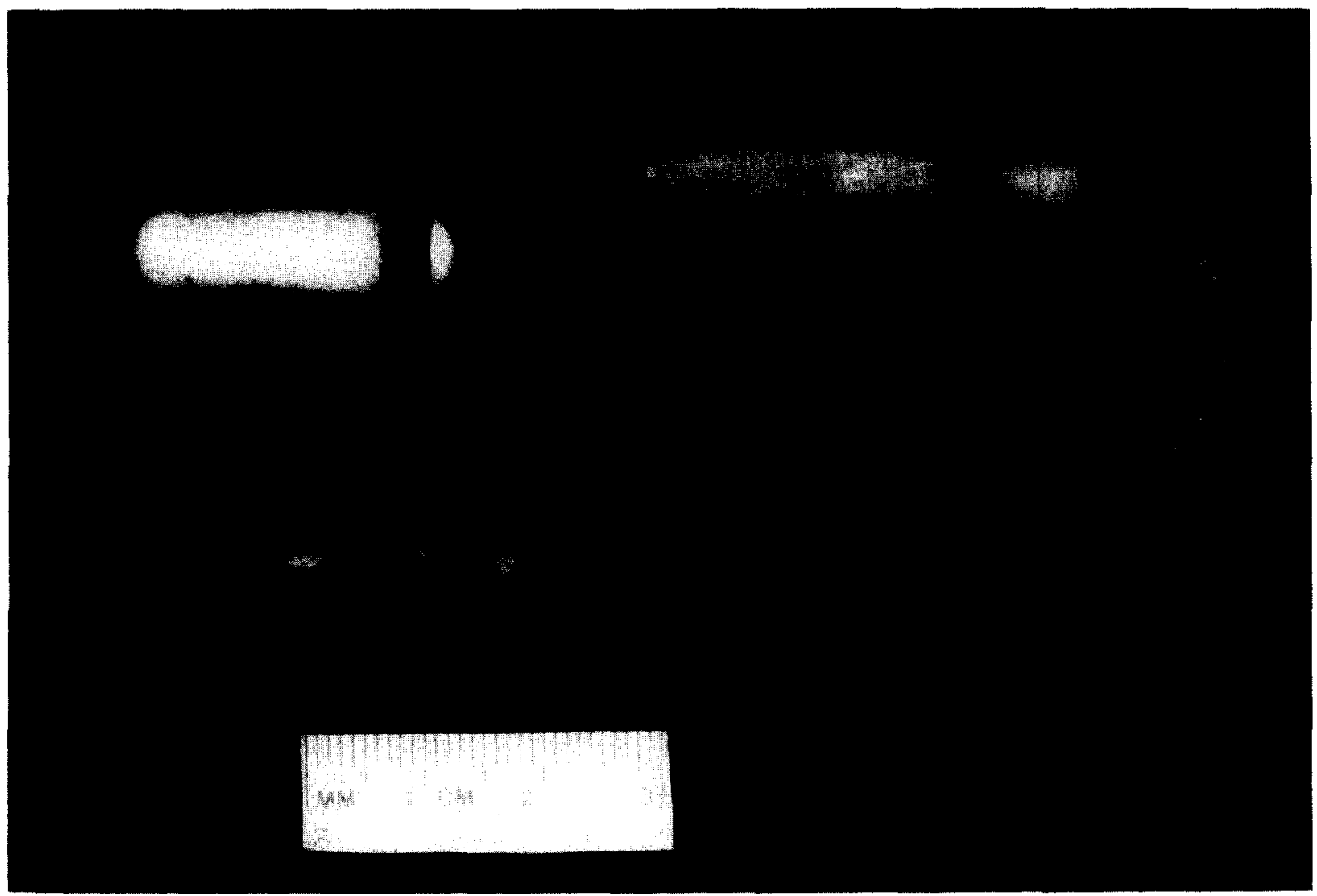

Fig. 2. A chronic infusion apparatus ready for implantation. The pipette tip sleeve shown was securely fitted to the probe prior to implantation and cemented in place upon completion of the procedure. 
threaded through an 8-cm length of Micro-line (TM) 1810 tubing.

The narrow end of a $200-\mu \mathrm{l}$ pipette tip is cut so that a 10-mm length of Micro-line 1850 tubing will fit firmly in place with $4 \mathrm{~mm}$ extending from the pipette tip (see Fig. 1). The 26-ga stainless steel tubing with attached dialysis fiber is then carefully guided down the pipette tip and through the Micro-line tubing until the PE20 tubing contacts the Micro-line tubing. The pipette tip is filled with epoxy and allowed to dry overnight.

Step 3. To attach the collection tube, use a 24-mm length of 20 -ga thin wall stainless steel tubing cleaned but not acid etched. Micro-line 1850 tubing $(6 \mathrm{~cm})$ is attached to one end. Using suture silk, a 0.4-ml sample tube is tied to the stainless steel tubing. The collection tube top is removed and pierced from the inside out with a 30-ga hypodermic needle. The exposed fused silica is threaded from the probe into the needle and drawn through the collection tube cap with the needle. The cap is attached to the outlet tubing using Devcon 5-min epoxy. Five minute epoxy is also applied to secure the collection tube thread and Micro-line 1850 to the stainless steel tubing. When the epoxy has dried, the stainless steel tubing (with the attached collection tube) is attached to the PE20 inlet tubing. If working properly, fluid will appear at the exposed end of the fused silica (and nowhere else) when slowly infused through the apparatus.

A properly flowing unit is ready for use. The unit should be filled with the appropriate solution. The collection tube is capped, making sure to allow space between the end of the fused silica and the bottom of the tube so that flow is unobstructed. The upper portion of the collection tube should be pierced with a needle to allow air release during solution collection. An Alzet minipump is attached after activation according to the manufacturer's instructions and the system is operating.

\section{In vitro application}

Chronic dialysis probes were filled with a solution of $40 \mathrm{mM}$ QUIN in $0.1 \mathrm{M}$ phosphate buffered $0.9 \%$ saline (PBS; pH 7.4). Alzet minipumps were filled with a solution of $40 \mathrm{mM}$ QUIN containing
2 nmol [ $\left.{ }^{3} \mathrm{H}\right] \mathrm{QUIN}$ (specific activity: $8.0 \mathrm{Ci} / \mathrm{mmol}$ ). Pumps were incubated for $20 \mathrm{~h}$ in PBS at room temperature, after which time they were attached to the units and placed in individual $25-\mathrm{ml}$ beakers filled with PBS. Beakers with pumps in them were covered with parafilm. The dialysis probes extended out of the beakers and were submerged in different beakers containing 20-ml PBS. All beakers were placed in a water bath maintained at $37^{\circ} \mathrm{C}$ for 22 days. Each day PBS from the probe beakers was sampled for tritium content and probes were transferred to a new beaker containing 20-ml PBS.

Duplicate $100-\mu 1$ aliquots from each beaker were counted using Biosafe scintillation cocktail $(5 \mathrm{ml})$ in a Beckman LS8100 scintillation counter. When the 3 units ceased delivery of $\left[{ }^{3} \mathrm{H}\right] \mathrm{QUIN}$, liquid in the connecting tubing and collection vial was collected and remaining radioactivity was measured.

\section{In vivo application}

Animals (25 adult rats) were anesthetized with pentobarbital $(30 \mathrm{mg} / \mathrm{kg})$ supplemented with methoxyfluorane. For each rat, a stainless steel cannula ( $8 \mathrm{~mm}, 21-\mathrm{ga})$ was implanted through the skull aimed at the right striatum (stereotaxic coordinates: $0.5 \mathrm{~mm}$ anterior, $2.6 \mathrm{~mm}$ lateral from bregma; $1 \mathrm{~mm}$ ventral from skull surface). The cannula was secured in place with dental acrylic and kept patent with a stylet. The chronic dialysis apparatus was inserted 10 days later under anesthesia as described above. Osmotic minipumps (lot no. 51943, mean pumping rate $0.55 \mu \mathrm{l} / \mathrm{h}$, mean fill volume $225 \mu 1$ ) and collection tubes were secured subcutaneously through a small incision in the back of the neck. Each dialysis probe was sleeved in a section cut from a $200-\mu 1$ pipette tip (Fig. 1). A dialysis apparatus containing either $40 \mathrm{mM}$ QUIN or vehicle (PBS) was inserted through the guide cannula. Dental acrylic was used to secure the outer pipette sleeve. During the dialysis period, several animals disconnected either the inlet or outlet tubing. These animals were anesthetized and the tubing was reattached. Infusion probes and pumps were removed after 4 weeks. 


\section{Histology}

All animals were given an overdose of pentobarbitol and perfused through the heart with PBS followed by $4 \%$ paraformaldehyde in $0.1 \mathrm{M}$ sodium phosphate buffer. Brains were post-fixed overnight at $40{ }^{\circ} \mathrm{C}$ and cryoprotected in $20 \%$ sucrose phosphate buffer. Coronal sections (40 $\mu \mathrm{m}$ ) were collected on a freezing microtome and stained with cresyl violet.

\section{Materials}

The following items were used for construction of the chronic dialysis apparatus: stainless steel tubing, 20- and 26-ga thin wall (Small Parts Inc., Miami, FL); dialysis fiber (regenerated cellulose hollow fiber) MWCO 6000 (stock no. 132275, Spectrum Medical Industries, Houston, TX); epoxy, 5-min and 2-ton (Devcon, Danvers, MA); fused silica capillary tubing, $75 \mu \mathrm{m}$ inner diameter $\times 150 \mu \mathrm{m}$ outer diameter (Polymicro Technologies, Phoenix, AZ); polyethylene tubing, PE20 (A-M Systems, Everett, WA); Micro-line (TM) 1810 and 1850 tubing (Cole-Parmer, Chicago, IL); and Alzet minipumps, model 2002 (Alza Corp, Palo Alto, CA).

For testing the apparatus, quinolinic acid (Sigma, St. Louis, MO) and $\left[{ }^{3} \mathrm{H}\right]$ quinolinic acid (NEN-Dupont, Bannockburn, IL) were used. For in vitro testing, samples containing $\left[{ }^{3} \mathrm{H}\right]$ QUIN were mixed with Biosafe scintillation cocktail (Research Products International, Mt. Prospect, IL).

\section{Results}

\section{In vitro dialysis with quinolinic acid}

$\left[{ }^{3} \mathrm{H}\right]$ QUIN diffused out of all dialysis probes for 14-17 days (Fig. 3). After the pumps containing $\left[{ }^{3} \mathrm{H}\right]$ QUIN were attached to the inlet tubing, it took 1-2 days for the $\left[{ }^{3} \mathrm{H}\right] \mathrm{QUIN}$ to reach the dialysis probe. By day 21 radioactivity levels in all 3 receptacle beakers were negligible. After that time, the percent total $\left[{ }^{3} \mathrm{H}\right] \mathrm{QUIN}$ was determined by subtracting the sum of the daily amounts collected from the total amount of $\left[{ }^{3} \mathrm{H}\right]$ QUIN

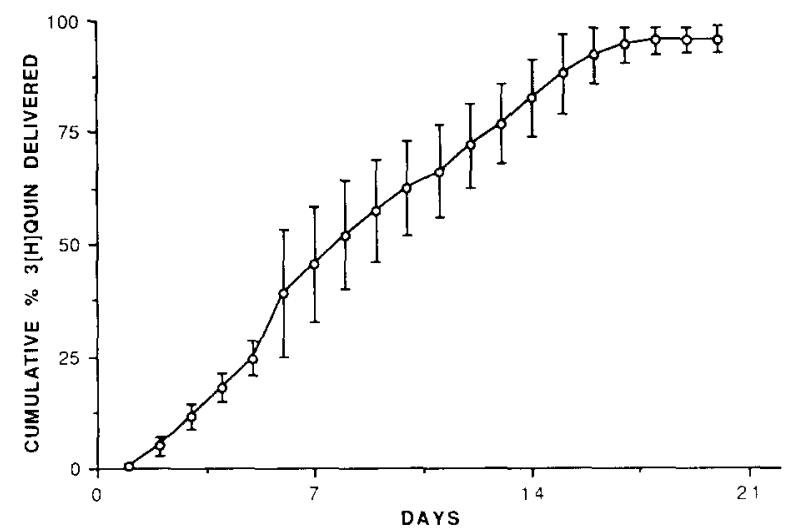

Fig. 3. Mean cumulative percent delivery of 3[H]QUIN over a period of 21 days. Mean cumulative delivery for the 3 pumps on day 21 was $95.3 \%$.

(sum of the daily amounts plus amount remaining in the apparatus at the end of the study). The percent efficiency of $\left[{ }^{3} \mathrm{H}\right] \mathrm{QUIN}$ dialysis from the units was: unit 1, 98.1; unit 2, 94.6; and unit 3, 93.2.

\section{In vivo dialysis of quinolinic acid}

Damage caused by QUIN dialysis consisted of atrophy of the right striatum. There was aiso atrophy of surrounding structures in some animals, but to a lesser extent (Fig. 4a). Animals that received vehicle had only local damage from the insertion of the dialysis probe (Fig. 4b). Animals from both the QUIN and vehicle groups remained healthy throughout the duration of the dialysis period and for the several weeks they were allowed to survive after removal of the apparatus. All pumps were inspected visually upon removal and found to be empty.

\section{Discussion}

A system has been described for chronic intracranial drug administration in the rat using a modified in vivo microdialysis probe in combination with the Alzet model 2002 osmotic minipump. The results presented demonstrate that this system can be used for the chronic administration of QUIN with minimal non-specific damage. Each pump delivered by osmosis the solute contained 
in approximately $225 \mu \mathrm{l}$ of solution over a period of 19-20 days, when tested in vitro. The dialysis units were uniform in function, $>93 \%$ of the $\left[{ }^{3} \mathrm{H}\right] \mathrm{QUIN}$ initially loaded into the minipump diffused out of the probe during operation. This same apparatus design was also successfully tested in vivo. Histological examination indicated that QUIN produced damage throughout the striatum.

When tested in vitro, $\left[{ }^{3} \mathrm{H}\right] \mathrm{QUIN}$ was not detected for the first 2 days of sampling. Since the inlet tubing was not filled with $\left[{ }^{3} \mathrm{H}\right] \mathrm{QUIN}$, the first 2 days of testing constituted a time course for solution (approximately $16 \mu \mathrm{l}$ ) to traverse the inlet tubing. Specifications from the pump lot used for in vitro tests predicted approximately 17 days of pumping. This time was lengthened by 1-2 days for in vitro testing, possibly due to continued diffusion of fluid contained in the inlet tubing after pump flow had ceased. Since the Alza corporation reports that the rate of infusion for in vivo pumps is within $5 \%$ of in vitro rate. the current apparatus design likely delivers drug over a similar time period in tissue.

For in vivo analysis of this apparatus the dose of QUIN tested produced extensive destruction of the striatum. Brain tissue from control animals that received PBS showed no gliosis in excess of that expected from probe insertion. Glial fibrillary acidic protein staining of a separate group of similarly treated animals also indicated that gliosis in the region of the probe was negligible (unpublished results). These results differ from Benveniste and Diemer (1987) who reported excessive gliosis surrounding chronically implanted dialysis fibers. At least two important methodological differences between that report and the present experiment may account for the contrasting results. Benveniste and Diemer (1987) reported excessive glial formation surrounding inactive acrylic dialysis fibers. In the present study, continuous dialysis was performed using regenerated cellulose dialysis fibers. Benveniste and Diemer (1987) reported astrocyte processes ex-

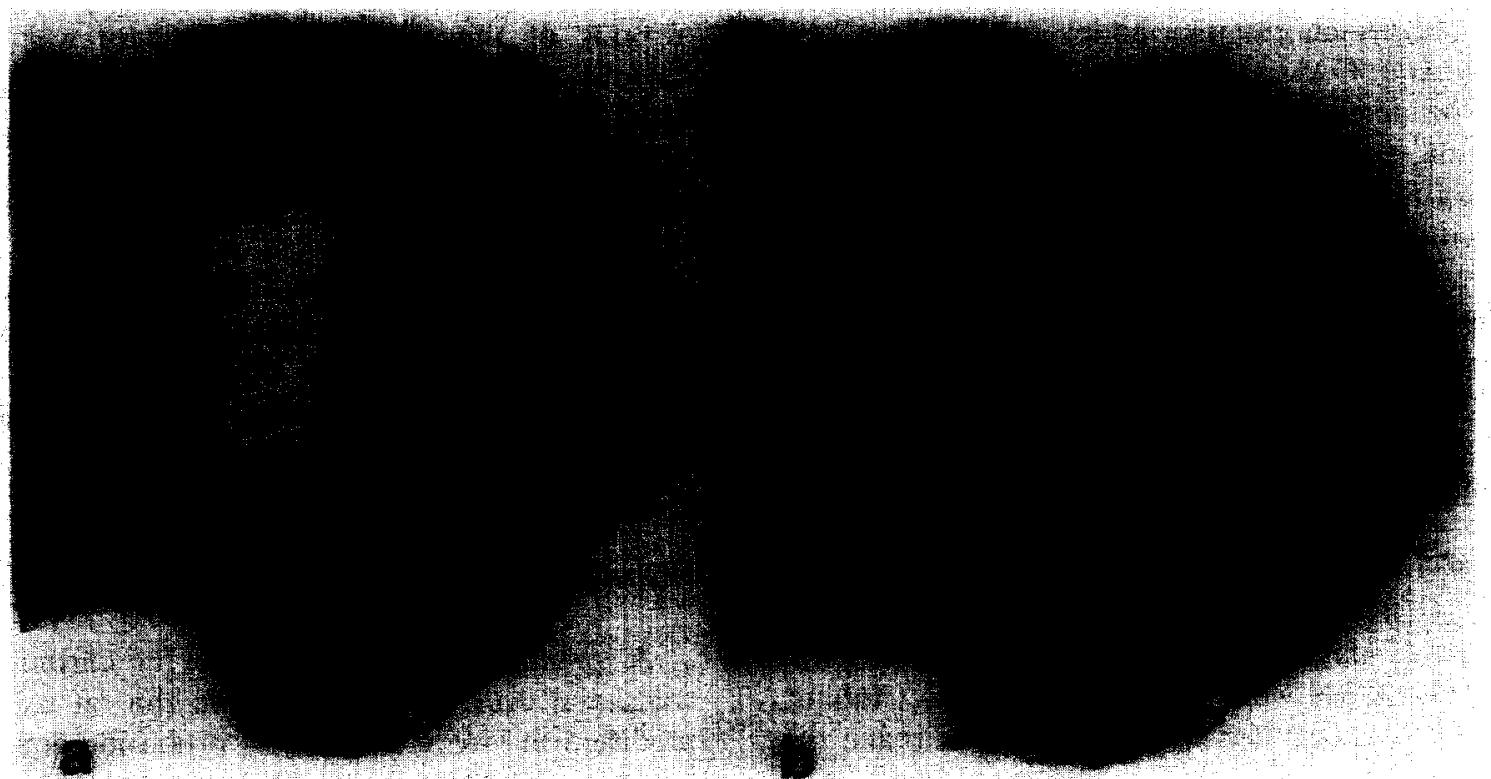

Fig. 4. a: cresyl violet stained $40 \mu \mathrm{m}$ section from the right striatum of a rat that received $200 \mu 1$ of a $40 \mathrm{mM}$ solution of QUIN over approximately 3 weeks. Marked atrophy of the striatum is apparent. The enlarged ventricle appears to have resulted from shrinkage of surrounding tissue. b: cresyl violet stained $40 \mu \mathrm{m}$ section from the right striatum of a control rat that received $200 \mu \mathrm{l}$ of PBS over approximately 3 weeks. Damage appeared non-specific and confined to the area immediately surrounding the probe cannula. 
tending into the fiber wall. Continuous dialysis may have reduced innervation within and around the fiber. In addition, the use of regenerated cellulose, rather than acrylic fibers, may also have reduced gliosis in the present experiment. In their study, Benveniste and Diemer (1987) noted that tissue reactions may have been caused by specific materials introduced into the brain, rather than the method by which they were introduced.

Use of chronic dialysis for administration of drugs may increase the reliability of administration, while decreasing non-specific tissue damage. Solutions that are pressure injected through an intracranially implanted cannula are assumed to enter the brain as a spherical drop (Rice et al., 1985). Variation in drop size directly influences the rate and extent of drug diffusion. Efficiency of binding, reuptake and degradation of infused substances may in turn be influenced by the rate of diffusion. Thus, variations in drop size, resulting from pressure injection delivery, may ultimately produce variability in the diffusion area, and in the effect of the infused drug. In addition, the use of injection cannulae results in point source delivery. These limitations apply to previous efforts to induce neural degeneration with excitotoxins using Alzet pumps attached to chronically implanted cannulae. The striatal injury induced with chronic infusion has resulted in spherical areas of necrosis limited to the area surrounding the cannula tip (Mangano and Schwarcz, 1983; McBean and Roberts, 1983; Rieke et al., 1989).

An additional advantage to this system is the potential to monitor proper operation throughout a period of chronic drug administration. A properly functioning unit continuously increases the volume of fluid in the collection tube. Although in the present in vivo study collection tubes were implanted subcutaneously, it is feasible that the collection tube could be secured to the skin surface. Previously, chronic infusion could be monitored only by inclusion of a radioactive substance that was then measured in daily urine samples (Mangano and Schwarcz, 1983).

By using osmosis across a concentration gradient with a semipermeable dialysis fiber, no solution drop is formed. In fact, there is no net change in fluid volume in the brain during the course of dialysis. The length of this fiber may be varied to increase or decrease the dialysis area. Furthermore, any organ that can be penetrated by the dialysis probe is a potential site for use of this system. In conclusion, we have developed a simple and inexpensive technique for administration of drugs into brain parenchyma with substantial advantages over previously used techniques.

\section{Acknowledgements}

The authors would like to thank Kevin Kaatz and Lily $\mathrm{Hu}$ for their technical assistance. This project was supported by The Hereditary Diseases Foundation, and Dr. Becker's USPHS grants NS22157 and NS25662. Dr. Becker is supported by a Research Career Development Award NS01056). Dr. Albin is supported by a Clinical Investigator Development Award (NS01300).

\section{References}

Benveniste, H. and Diemer, N.H. (1987) Cellular reactions to implantation of a microdialysis tube in the rat hippocampus. Acta Neuropathol. (Berl.), 74: 234-238.

Evans, B.K., Armstrong, S., Singer, G., Cook, R.D. and Burnstock, G. (1975) Intracranial injection of drugs: comparison of diffusion of 6-OHDA and guanethidine. Pharmacol. Biochem. Behav., 3: 205-217.

Hargraves, R. and Freed, W.J. (1987) Chronic intrastriatal dopamine infusions in rats with unilateral lesions of the substantia nigra. Life Sci., 40: 959-966.

Mangano, R.M. and Schwarcz, R. (1983) Chronic infusion of endogenous excitatory amino acids into rat striatum and hippocampus. Brain Res. Bull., 10: 47-51.

McBean, G.J. and Roberts, P.J. (1984) Chronic infusion of L-glutamate causes neurotoxicity in rat striatum. Brain Res., 290: 372-375.

Mendel, V.E., Benitez, R.R. and Tetzke, T.A. (1988) Human satietin: rapid development of tolerance and its specificity to feeding behavior in rats. Pharmacol. Biochem. Behav. 31: 21-26.

Nielsen, E.B. (1981) Rapid decline of stereotyped behavior in rats during constant one week administration of amphetamine via impanted ALZET osmotic minipump. Pharmacol. Biochem. Behav., 15: 161-165.

Rice, M.E., Gerhardt, G.A., Hierl, P.M., Nagy, G. and Adams. R.N. (1985) Diffusion coefficients of neurotransmitters 
and their metabolites in brain extracellular fluid space. Neuroscience, 15: 891-902.

Rieke, G.K., Smith, J., Idusuyi, O.B., Semenya, J., Howard, R. and Williams, S. (1989) Chronic intrastriatal L-pyroglutamate: neuropathology and neuron sparing like Huntington's disease. Exp. Neurol., 104: 147-154.

Rivier, C. and Vale, W. (1989) Immunoneutralization of endogenous inhibin modifies hormone secretion and ovulation rate in the rat. Endocrinology, 125: 152-157.

Robinson, T.E. and Camp, D.M. (1991) The feasibility of repeated microdialysis for within subjects design experi- ments: studies on the mesostriatal dopamine system. In T.E. Robinson and J.B. Justice (Eds.), The Neurosciences. Elsevier, Amsterdam, pp. 189-234.

Sasamura, H., Suzuki, H., Kato, R. and Saruta, T. (1990) Effects of angiotensin II, ACTH, and KCL on the adrenal renin-angiotensin system in the rat. Acta Endocrinol. (Copenh.), 122: 369-373.

Sendelbeck, S.L. and Urquhart, J. (1985) Spatial distribution of dopamine, methotrexate and antipyrine during continuous intracerebral microperfusion. Brain Res.. 328: $251-$ 258. 